\title{
A Motivational Approach to Support Healthy Habits in Long-term Child-Robot Interaction
}

\author{
Raquel Ros ${ }^{* 1}$, Elettra Oleari ${ }^{\dagger 2}$, Clara Pozzi ${ }^{\ddagger 2}$, Francesca Sacchitelli ${ }^{\S 2}$, Daniele Baranzini ${ }^{\boldsymbol{} 2}$, \\ Anahita Bagherzadhalimi ${ }^{\| 2}$, Alberto Sanna ${ }^{* * 2}$ and Yiannis Demiris ${ }^{\dagger \dagger 1}$ \\ ${ }^{1}$ Personal Robotics Laboratory, Department of Electrical and Electronic Engineering, \\ Imperial College London, Exhibition Road, SW7 2BT, London UK \\ ${ }^{2}$ Fondazione Centro San Raffaele, via Olgettina 60, 20132, Milano, Italy
}

\section{Abstract}

We examine the use of role-switching as an intrinsic motivational mechanism to increase engagement in long-term child-robot interaction. The present study describes a learning framework where children between 9 and 11-years-old interact with a robot to improve their knowledge and habits with regards to healthy life-styles. Experiments were carried out in Italy where 41 children were divided in three groups interacting with: $(i)$ a robot with a role-switching mechanism, (ii) a robot without a role-switching mechanism and (iii) an interactive video. Additionally, a control group composed of 43 more children, who were not exposed to any interactive approach, was used as a baseline of the study.

During the intervention period, the three groups were exposed to three interactive sessions once a week. The aim of the study was to find any difference in healthy-habits acquisition based on alternative interactive systems, and to evaluate the effectiveness

\footnotetext{
*raquel.ros.espinoza@gmail.com

†oleari.elettra@hsr.it

${ }^{\ddagger}$ pozzi.clara@hsr.it

§sacchitelli.francesca@hsr.it

ฯ baranzini.daniele@hsr.it

" bagherzadhal.anahita@hsr.it

**sanna.alberto@hsr.it

$\dagger^{\dagger}$ y.demiris@imperial.ac.uk
}

of the role-switch approach as a trigger for engagement and motivation while interacting with a robot. The results provide evidence that the rate of children adopting healthy habits during the intervention period was higher for those interacting with a robot. Moreover, alignment with the robot behaviour and achievement of higher engagement levels were also observed for those children interacting with the robot that used the role-switching mechanism. This supports the notion that role-switching facilitates sustained long-interactions between a child and a robot.

\section{Introduction}

Motivation plays an important role in humans by promoting adherence to therapy treatment, improving task performance or encouraging behavioural change. Motivation could be either intrinsic or extrinsic. Intrinsic motivation originates from the agent's internal processes when the agent is doing something which is inherently interesting or enjoyable [37]. Intrinsic motivation is mostly influenced by three factors: enjoyment, indirect competition and the (user dependent) optimal level of challenge for the task. On the other hand, Extrinsic motivation comes from sources external to an agent. For example, incentives such as money, prizes or grades are considered as extrin- 
sic motivators. Intrinsic motivation has been demonstrated to be more effective for long-term tasks and behavioural change, whereas extrinsic motivation has shown to be more effective for short-term task compliance [12].

Positive encouragement and strong engagement are key factors for increasing intrinsic motivation in humans to perform a task or achieve a behavioural change, such as acquiring healthy habits [44]. Within this context, intrinsic motivation is critical in children in behavioural change scenarios, as they need to learn, understand, and maintain a habit for a long period of time.

Investigating the capability of robots to motivate children to learn new tasks, or develop specific habits, has been of major interest in recent years. This is true particularly in contexts where it is promoting health and providing learning support (such as hospitals and schools) [9]. In the field of Child-Robot Interaction (CRI), robots can promote children's intrinsic motivation in multiple ways, especially in the form of positive reinforcement and encouragement.

The present work aims to investigate an educational framework, guided by a humanoid robot, to intrinsically motivate children of school age to adopt a healthy and active lifestyle. The framework centres around a dance activity, creative dance [40], in which children between 9 and 11-years-old interact with a robotic dance tutor once a week for three consecutive weeks. During these dance sessions, where dance movements are related to healthy theme concepts, they discover the most important nutritional and physical activity-related guidelines to achieve a healthy lifestyle. Moreover, our work is inspired by the learning by teaching approach proposed by Tanaka et al. [42], where children act as tutors, and the robot as the apprentice. Thus, we propose the use of a role reversal between child and robot (hereafter referred as role-switching) throughout the sessions. Within each session the child will exchange roles, acting first as an apprentice (or follower) and then as a tutor (or leader).

In the present study we evaluate the motivational effectiveness of our approach in terms of knowledge gained (also referred as awareness in the text) and improvement of habits for the participating children, and to assess their level of engagement in the activity proposed. To this end, we compare the same learning framework, but with two different types of tutors: either a humanoid robot or an interactive video. This experimental design allows us to evaluate the effectiveness of the robot-based model against a comparably entertaining technology for children. We conducted the relevant quantitative and qualitative analyses in order to obtain evidence regarding the validity of our research hypotheses. The study has been designed and conducted by a multidisciplinary team of experts from social robotics, psychology and nutrition.

The paper is structured as follows: we first describe related research, particularly from ChildRobot Interaction, motivational support and empathetic agents (Section 2). We then present the interactive framework structure we have designed (Section 3) followed by a brief description of the robot architecture (Section 4). The experimental procedure (Section 5) along with its results are introduced next (Section 6). The paper concludes with a discussion on the insights gained and future perspectives of this research (Sections 7 and 8 respectively).

\section{Related Work}

For more than a decade research on Human-Robot Interaction (HRI) has been exploring the features that could regulate and support a successful and natural interaction between humans and robots [13]. Numerous studies have revealed the importance of incorporating social skills into robots to achieve such an ambitious goal $[11,39,38,7]$, specifically in the longterm $[1,6,4,19,14]$. A survey on social robots for long-term interaction can be found in the work of Leite et al [23].

More recently, the scientific community has increasingly turned its attention to the study of childrobot interactions (CRI) [24, 16, 41, 3]. While children may have a natural predisposition to easily engage with robots due to their novelty, it is also very challenging to sustain such an engagement over a long period of time when the novelty effect diminishes $[22,35]$. Therefore this work addresses the need for 
a deeper understanding of motivational mechanisms to achieve long-term engagement.

Researchers have focused their studies on a wide range of applications such as the learning and promotion of knowledge. Three common learning styles (i.e. learning from lectures, learning from performing cooperative tasks and self-directed learning) were implemented into the ASIMO humanoid robot to see if children can learn from robots in a tabletop scenario $[30,28]$. The effects of adopting certain general features such as a monotone robot-like voice and human-like voices were also compared, suggesting that human features increase children social behaviour. With this in mind, in our work the robot is provided with human-like behavioural cues to enhance social interaction.

A learning by teaching approach is introduced in [42], where learning is expected to occur as children take care or instruct robots on English verbs. The results provide insight on the efficiency of the approach, where children achieved higher scores when learning with a care-receiving robot than without, and also highlight different natural forms of teaching methods that children used while interacting with the robot.

Another research line proposes the use of active learning, i.e. learning promoted by interacting with one's environment as opposed to dictated teaching [26]. In this approach, the user is able to actively participate and influence on the way the activity is presented and therefore, they not only obtain and retain more information, but also become more engaged in the task.

Our research is inspired by a combination of these approaches, where the child plays the role of both the apprentice and the tutor throughout the sessions.

Another area of exploration is that of acquisition and improvement of physical skills and cognitive skills. A robotic weight loss coach is introduced in [18] where the need for long-term, supportive care in bariatric settings is essential. However, the work mainly focuses on verbal support from a robot and does not extend to the robot's physical capabilities to actively motivate the user as our work does. In [12], a robot that monitors the performance of adults during a combined cognitive and physical task is presented. The goal is to provide motivation to the user to com- plete the task and to improve task performance.

In our work, while we also focus on motivation to fulfil the task, we are more interested on knowledge and habit improvements rather than improving task performance.

The application of robotics for motivation and selfefficacy in therapeutic contexts has been investigated in several contexts. In the diabetic context, Lewis and Cañamero [25] focus their work on the affective and social aspects of the interaction between a robot toddler and diabetic children to support the development of self- efficacy. Wada et al use PARO, a seal robot, for therapy on adult patients suffering dementia. Results show that the mood of patients and elderly people improved, making them more active and communicative with each other and their caregivers as well as reducing stress. Moreover, long-term interaction was investigated and effects of interaction lasted for more than a year [47, 45, 46]. Dautenhahn and her team have mainly focused on the development of interactive games for autistic children helping them to develop and increase their communication and social interaction skills. Turn-taking and imitation games related to education and therapeutic objectives are presented to children to study the effects of touch [34], as well as long-term exposure to robots [33].

In this work we adopt a hybrid approach to increase the children's engagement and motivational support of children: (i) part of the sessions are guided by the robot in a tutor-pupil approach, where the learning concepts are first introduced and (ii) whilst the remainder of the session is led by the children in a peer-to-peer approach, where knowledge retention is expected.

\section{Interactive Learning Frame- work}

The purpose of our research is to demonstrate the intrinsic motivational effectiveness of a robot-based interactive learning approach, within a creative dance activity context. Dance was chosen as the reference activity because it is widely considered entertaining, 


\begin{tabular}{|c|c|c|c|c|c|c|}
\hline $\begin{array}{l}\text { Session } \\
\text { number } \\
\& \text { title }\end{array}$ & $\begin{array}{l}\text { Dance } \\
\text { con- } \\
\text { cept }\end{array}$ & Description & Instances & $\begin{array}{l}\text { Pyramid } \\
\text { level }\end{array}$ & $\begin{array}{l}\text { Examples } \\
\text { of food }\end{array}$ & $\begin{array}{l}\text { Examples } \\
\text { of physical } \\
\text { activity }\end{array}$ \\
\hline $\begin{array}{lr}\text { 1: } & \text { 'What } \\
\text { should } & \text { we } \\
\text { eat/do } & \text { every } \\
\text { day' } & \end{array}$ & shapes & $\begin{array}{l}\text { no motion, } \\
\text { shape represen- } \\
\text { tation with the } \\
\text { body }\end{array}$ & $\begin{array}{l}\text { big, small, } \\
\text { straight, } \\
\text { curved, } \\
\text { spiky }\end{array}$ & 1 & $\begin{array}{l}\text { cereals, wa- } \\
\text { ter, vegeta- } \\
\text { bles, fruits }\end{array}$ & $\begin{array}{l}\text { walking, } \\
\text { using stairs, } \\
\text { outdoor play }\end{array}$ \\
\hline $\begin{array}{l}\text { 2: 'What } \\
\text { should we } \\
\text { eat/do sev- } \\
\text { eral times a } \\
\text { week' } \\
\text { 3: }\end{array}$ & $\begin{array}{l}\text { sudden } \\
\text { actions }\end{array}$ & $\begin{array}{l}\text { quick move- } \\
\text { ments with any } \\
\text { body part }\end{array}$ & $\begin{array}{l}\text { jump, roll, } \\
\text { fidget, swing }\end{array}$ & 2 & $\begin{array}{l}\text { fish, } \\
\text { legumes, } \\
\text { nuts }\end{array}$ & $\begin{array}{l}\text { cycling, } \\
\text { swimming, } \\
\text { tennis }\end{array}$ \\
\hline $\begin{array}{l}\text { should we } \\
\text { eat/do rarely } \\
\text { or try to } \\
\text { avoid, }\end{array}$ & $\begin{array}{l}\text { smooth } \\
\text { actions }\end{array}$ & $\begin{array}{l}\text { slow movements } \\
\text { with any body } \\
\text { part }\end{array}$ & $\begin{array}{l}\text { balance, } \\
\text { extend, pull, } \\
\text { push }\end{array}$ & 3 & $\begin{array}{l}\text { meat, eggs, } \\
\text { pizza } \\
\text { sweets, fizzy } \\
\text { drinks, chips }\end{array}$ & $\begin{array}{l}\text { stretching, } \\
\text { push ups, } \\
\text { gardening } \\
\text { watching } \\
\text { TV, } \\
\text { videogames }\end{array}$ \\
\hline
\end{tabular}

Table 1: Summary of the three sessions' structure, indicating the dance concepts addressed per session number and their link with the Levels of the pyramids explored. Session 3 covers two Levels of the pyramids, i.e. Levels 3 and 4 . Level 4 of the pyramids does not have a link to dance motions. Therefore, no concepts are reviewed at this stage of the session. 
and it allows children to easily engage due to the involvement of movement, which in turn increases enjoyment $[14,5,1,43]$.

The desired result is an interactive fun learning environment that can be used as supportive tool for teachers, where children can receive the right stimuli to express their creativity and be encouraged to continue the educational process over a period of time. In expressing their creativity, children internalise concepts related to healthy habits, explained in the various sessions, and become aware of the importance of their application in daily choices.

We refer in particular to creative dance as a form of dance where the goal is to explore the body's movements based on a set of general guidelines (i.e.: movement concepts). On the one hand, creative dance provides elements of dance foundations, movement vocabulary and locomotive skills. On the other, it promotes creativity which motivates pupils to expand and extend movement range at their own rhythm through different stimuli, such as music, emotions, visual resources and observing the creativity of others [40].

The proposed learning framework adopts a relational model [36], where dance concepts are linked to health theme concepts. Through this model children can acquire knowledge on dance movements explained by the dance tutor and, at the same time, learn or reinforce other learning topics (in this work, adopting healthier life-styles). We structure the contents of the sessions based upon the study of two pyramids: the Food pyramid $[10,31]$ and the Physical Activity pyramid [29]. These schemes represent the frequency in which the different food and activities should be eaten and performed respectively, where bottom levels correspond to higher frequency (i.e. eat or perform quite often) and top levels to lower frequency (i.e. eat or perform rarely or even avoid). Figure 1 details the contents of the two pyramids used in the current framework.

The overall dance framework is divided in three sessions, each linking a dance concept with one level of each pyramid (except for the last session where Levels 3 and 4 of the pyramids are covered). The themes explained during the sessions were specifically designed to cover the national and European guidelines [10,31]
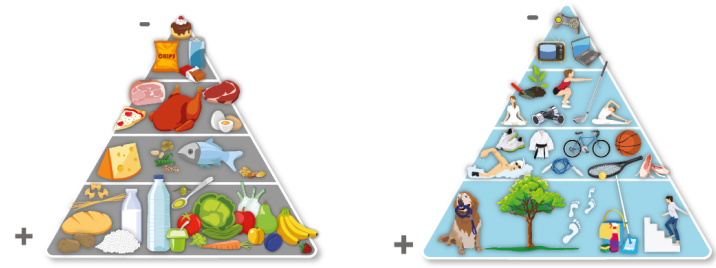

Figure 1: Food (left) and physical activity (right) pyramids describing the recommended weekly frequency of food intake and activity performance.

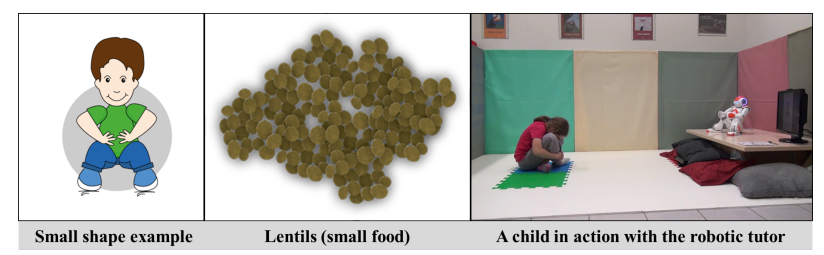

Figure 2: Example of the relational-based model used in the sessions corresponding to the link between a small shape (left) and food (middle). (Right) The dance tutor, in this case the robot, asks the child to represent the shape of the food shown in the image.

on proper diet and physical activities that should be followed to achieve a healthier lifestyle. A detailed description of each session is provided in Table 1.

An example of the way in which the relational model works is as follows: the tutor goes through the small shape dance concept, which involves performing full or partial body motions where the body parts become as small as possible (e.g. a fist is a small shape of the hand). The tutor then asks children to think of a food which has this small attribute and to represent it with their body. Figure 2 depicts a child crouching on the floor to represent a lentil.

In the present study the dance tutor can either be represented by a humanoid robot or an interactive video to compare both approaches. The next section describes the system architecture for the robot-based approach. 


\section{Robot System Architecture}

The robot selected for this study is Nao, a humanoid robot from Aldebaran Robotics ${ }^{1}$. The Nao robot is almost $58 \mathrm{~cm}$ tall, weighs $5.2 \mathrm{~kg}$ and has a cartoonlike appearance which was considered especially suitable for use with children. Its 25 degrees of freedom allow smooth motions which are required especially for dance movements. LEDs are located in the head, eyes, ears, chest and feet. Two loudspeakers are located in the ears to play speech. It is also equipped with two cameras and a microphone. The robot's behaviour is autonomous although it requires the intervention of a Wizard of $\mathrm{Oz}$ only for perception (visual and speech inputs). However, the robot was presented to the children as fully autonomous, i.e. operating without human intervention.

Within this work, the robot's behaviour is twofold. On the one hand, it performs the activity acting as a tutor, guiding children through the different sessions. On the other hand, it plays the role of a motivator keeping children engaged in the task as long as possible, not only to finish it, but to repeat the encounter in future occasions. We describe both aspects in more detail next.

\subsection{Task performance}

The goal of the robot is to perform the dance activity in a structured way. Each session is comprised of the following stages:

1. Introduction: the goals of the current session are explained and a brief recap of the previous ones (if any) is given.

2. Warm-up: a fixed sequence of movements is performed to warm up the body in preparation for the session's exploration.

3. Exploration: dance concepts are visited one by one, providing examples and requesting children to create new ones to promote creativity.

4. Consolidation: dance concepts are linked to the pyramids through an adaptation of the musical

\footnotetext{
${ }^{1}$ www.aldebaran.com
}

chairs game. Children travel around the room (we will refer to this task later on the paper as the travelling task) using the dance concepts seen so far (e.g. walking in circles, jumping around, etc.) and stop when the music stops. Role-switching takes place at this point: either the robot asks a question and children have to answer (no role reversal -the robot continues leading the activity), or children ask a question and the robot has to reply back (role reversal case -children lead the course of the activity). These two variations correspond to two of the four conditions in this study, which will be described in detail later on. Moreover, during this stage, children are given an explanation on the importance of the different food types and physical activities in the corresponding pyramid level, as well as the frequency in which they should be eaten and performed.

5. Farewell: a brief summary of the session is given and children are encouraged to practice the concepts they have learned with different motivational hints.

\subsection{Motivational support}

Apart from the inclusion of the role-switching mechanism as a motivational tool, additional cues for enhancing social interaction are also included in the design of the robot's behaviour. These types of behaviours prevent the robot from being too static and therefore more human-like as suggested in $[8,16,7]$ and based on our findings in previous works [2]:

- Body cues: head movements, blinking eyes, spatial orientation of the robot;

- Verbal cues - relationship: robot's ability to express recognition and familiarity (e.g., using the child's name, referring to previous shared experiences);

- Verbal cues - motivation: specific motivational phrases such as: "Try to do more exercise even when you're at home, ok?", "I know it's difficult to avoid this food, but I'm sure you can do it". 
Moreover, an additional motivational element is introduced. Children have to sign a pact with robot where they commit to pursue two self-assigned goals related to healthy living. Further details are provided in Section 5.2.1.

\subsection{System Architecture}

Figure 3 illustrates the robot's system architecture used in this work (introduced in more detail in [35]). The implementation of the system was done using $\mathrm{Urbi}^{2}$, an open-source software platform to control robots:

- Dance Manager: controls the overall robot behaviour, implementing the different methodologies used in the stages of the sessions and the engaging behaviours described in the sections above.

- WoZ: provides external input whilst the perceptual components are completed. It allows for evaluation of children's performance and answers to questions. Additionally, it also permits the operator to re-position the robot, if necessary.

- Dance Move Manager: manages the library of dance moves and provides information to the Non-Verbal Behavior for their execution.

- User Model: in charge of handling the users' interaction history to enable adaptation in the decision process. Besides storing general information about the child (such as ID, name, age and gender), it also keeps track of the dance moves performed to inform the Dance Manager which dance move should be explored next.

- Verbal Behavior: responsible for the verbal output of the robot. The utterances are based on a text manually created. Variations of each message have been produced to avoid repetitive phrases.

- Text-To-Speech: in charge of the speech synthesis using the commercial Acapella TTS system prebuilt into the robot.

\footnotetext{
${ }^{2}$ www.urbiforge.org
}

- Non-Verbal Behavior: responsible for the nonverbal output of the robot.

- Body Motion: manages the robot's body movements.

- Blinking Eyes: controls the LEDs located in the robot's eyes to emulate blinking.

- Head Motion: controls the head movement when no other motion occurs.

\section{Methodology}

\subsection{Study design}

We designed a between-subject study in which the participants were divided into four groups, one per experimental condition:

- With Role Switching (WRS): participants interact with the robot and switch roles. During the first half of the sessions the robot plays the role of the leader, while the child plays the role of the follower. In the second half, the child becomes the leader, and the robot, the follower.

- Without Role Switching (W/O RS): participants always assume the role of the follower, while the robot assumes the role of the leader throughout all the sessions.

- Interactive Video $(V)$ : the robot is replaced by an interactive video. A set of scenes have been pre-recorded where an actor plays the role of the tutor providing instructions, explanations and feedback in the same way the robot does. During the interactions a wizard selects the next scene to show, based on a script and the current state of the interaction. Only visual feedback from the user is considered, so to maintain uniformity as compared to the activity's performance in the previous two groups. In this condition, the actor plays the role of the leader throughout the ses$\operatorname{sion}^{3}$. The aim is to evaluate the effectiveness

\footnotetext{
${ }^{3}$ This condition is analogous to the Without Role Switching condition, with the difference that children interact with a video instead of a robot.
} 
Preprint of paper to appear at the International Journal of Social Robotics (2016).

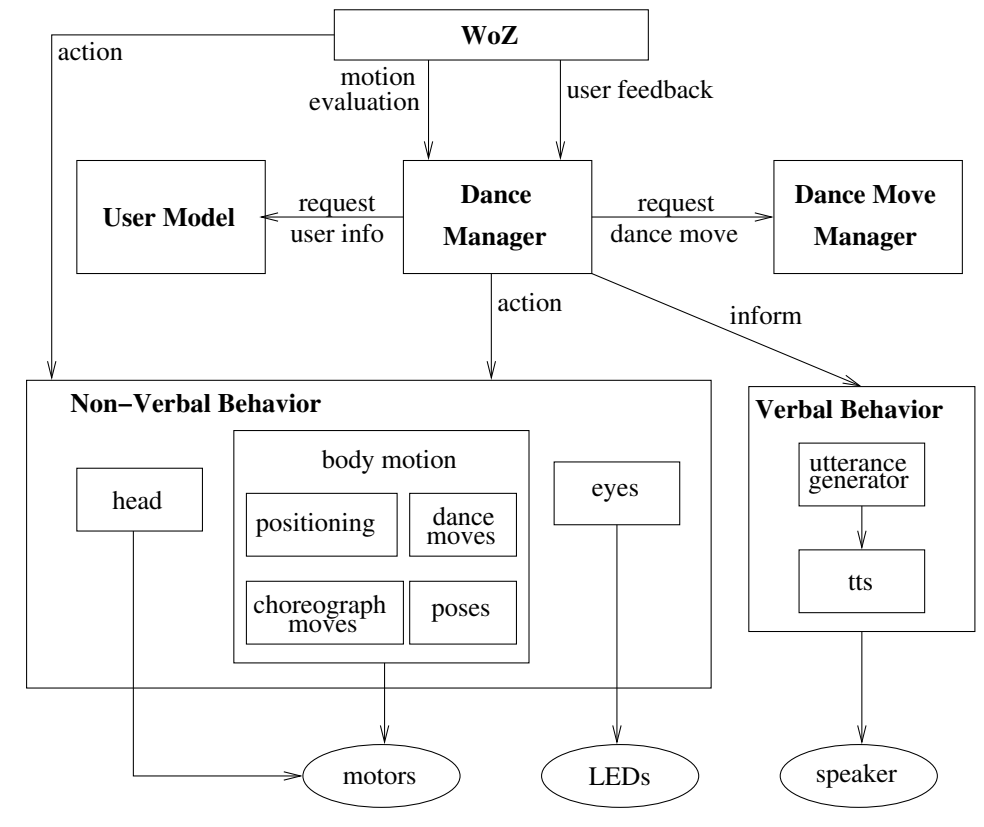

Figure 3: Diagram of the system architecture. Arrows represent the flow of information between components.

of a robot-based approach compared to another technology-based approach.

- Control $(C)$ : participants do not interact with neither a robot nor a video. This condition corresponds to the baseline of our study, to assess the impact of the proposed activity on acquisition of healthy habits compared to that acquired in their daily lives where no explicit activity is in place $^{4}$. Moreover, children in this group were located in a separate building from the rest of the children in the study, minimising the chances of talking to each other about the current study.

Table 2 shows a summary of the different condition groups and their corresponding number and demographics. Children that participated in any of the three first experimental conditions interacted with ei-

\footnotetext{
${ }^{4}$ We were informed by the teachers that, during the period in which the study took place, no explicit references to healthy habits were addressed in the involved classes. Therefore, if any information on this topic was provided to children, it had to be out of school lessons/activities.
}

\begin{tabular}{|c|c|c|c|c|c|c|}
\hline $\begin{array}{l}\text { Experimental } \\
\text { condition }\end{array}$ & Acronym & $\mathrm{N}$ & $\mathrm{M}$ & $\mathrm{F}$ & $\begin{array}{l}\text { Age } \\
9-10\end{array}$ & $\begin{array}{l}\text { Age } \\
10- \\
11\end{array}$ \\
\hline $\begin{array}{l}\text { With Role } \\
\text { Switching }\end{array}$ & $W R S$ & 14 & 6 & 8 & 6 & 8 \\
\hline $\begin{array}{l}\text { Without } \\
\text { Role Switch- } \\
\text { ing }\end{array}$ & $W / O R S$ & 14 & 7 & 7 & 7 & 7 \\
\hline Video & $V$ & 13 & 5 & 8 & 7 & 6 \\
\hline Control & $C$ & 43 & 23 & 20 & 22 & 21 \\
\hline
\end{tabular}

Table 2: The four study conditions acronyms and the related participants distribution ( $\mathrm{N}=$ total number; $\mathrm{M}=$ males; $\mathrm{F}=$ females).

ther the robot or the interactive video once a week for three weeks. Hereafter we will refer to these three groups as the study groups. Children in the Control group did not have any interaction with the system(s). 
Preprint of paper to appear at the International Journal of Social Robotics (2016).

\subsection{Measures}

We designed a set of measures to assess the following aspects: $(i)$ knowledge gained on healthy habits (awareness), i.e. nutrition and physical activity; (ii) impact on children's habits during the experimentation period; and (iii) engagement in the activity, with a special focus on child-robot interactions.

\subsubsection{Knowledge and Habits Measures}

To evaluate the possible changes in terms of acquired knowledge and habits improvements of the participating sample, two specific questionnaires on nutrition and physical activity were prepared: Knowledge (Q1) and Habits (Q2). Children filled in them right before and after the study period. Both questionnaires include open questions and True/False questions.

In addition to that, children were asked to selfassign two goals regarding healthy nutrition and/or physical activity to be maintained during the study. Participants had the chance to make their own choices among a list of proposed objectives specifically designed to be compared with their answers in the pre and post Habits questionnaire (Q2).

At the end of the study period, a third questionnaire, Behaviour Change (Q3), was addressed to the parents of the children in the study groups only. The purpose of this questionnaire was to analyse any change in behaviour, to discover if children shared with them what they learned during the study period, and their eventual influence on the family decisions regarding nutrition and physical activity.

\subsubsection{Engagement Measures}

After the last session, children in the study groups filled in a self-assessment questionnaire, the Engagement questionnaire (Q4). The aim of this questionnaire was to evaluate: $(i)$ children's enjoyment of the system and of the activity, based on Visual Analogue
Scale ${ }^{5}$ tools; (ii) their perception of the robot $^{6}$, based on multiple-choice selections of the following terms and adjectives: friend, puppy, adult, toy, computer and nice, funny, smart, fake, fragile, tender, affectionate respectively; and (iii), their reactions to the learning approach, i.e. what have they learned and what would they like to learn in the future with the system.

Moreover, three independent observers watched the video recording of each session to assess the level of perceived engagement of the child. To this end, a shortened version of the Positive Affect and Negative Affect Schedule - Child Form (PANAS-C) [20] questionnaire was used (a similar analysis was described in [32]). An expert psychologist of Ospedale San Raffaele identified the following single-word items to be evaluated by the observers on a 5-point Likert scale: interested, excited, happy, active, calm, frightened, jittery, nervous, sad and ashamed. The first five terms are related to positive affect and the last five, to negative affect. The aim is to evaluate any possible change in behaviour across the three sessions, depending on the study conditions.

All four questionnaires (Q1, Q2, Q3 and Q4) were designed with the support of experts in Nutrition Science and Psychology of Ospedale San Raffaele (Milan, Italy) and can be found in the Appendix.

\subsubsection{Observable Behaviour Measures}

We carried out a quantitative video coding analysis on the two groups that interacted with the robot (i.e., $W R S$ and $W / O R S$ conditions) to evaluate possible differences in children's behavioural cues during the interactions (similar coding schemes have been adopted in $[15,28,21])$. The selected cues were restricted to observable behaviour in order to avoid annotations based on personal interpretations of the situation (for example, smiling does not necessarily imply happiness).

Two coders labelled each video independently in a

\footnotetext{
${ }^{5} \mathrm{~A}$ pictorial representation of a 1-5 Likert scale through which respondents identify their answers, feelings or opinions [27].

${ }^{6}$ Children in the video condition $(V)$ did not filled in these questions.
} 
Preprint of paper to appear at the International Journal of Social Robotics (2016).

first round using the ELAN [48] software. In a second round a third coder compared the annotations and those which had different ${ }^{7}$ labels were re-evaluated to determine the final label. The inter coder reliability achieved was 0.86 .

We focused on the following observable behavioural cues:

- Visual contact with the robot: whether children's gaze is directed towards the robot or not, which was used to assess their attention.

- Verbal communication: speech occurrences classified as expected (answers to questions posed by the robot) or unexpected (children's free speech).

- Non-verbal communication: any type of body language used by children, such as nodding, thumbs up, pointing, etc. The aim is to assess how often they try to non-verbally communicate with the robot.

- Task related behaviour: actions (i.e.: body movements) related to the interactive framework to analyse the level of response of children to the sessions. During the sessions children are free to move and explore body motions as they want, guided by the robot's requests. We are thus interested in differentiating between expected and unexpected (proactive) behaviours. Moreover, we want to observe their response to the travelling task (part of the game played in the consolidation stage described in Section 4.1), where children are expected to move around the room. This task takes place during the stage where the roles can differ between conditions, and therefore, the corresponding children's performance can vary. From our previous works [36], we could notice that children who were not engaged in the task would not move (or very little) around the room.

\footnotetext{
${ }^{7}$ Few differences between annotations were found, and these were mainly due to human error, i.e. missing an observable behaviour, rather than disagreement on the classification of the behaviour.
}

\subsection{Participants}

The study was carried out in Italy, where 84 healthy children between 9 and 11-years-old were recruited. We worked with students in 4th and 5th grade from four different classes divided into four groups randomly assigned while balancing age and gender. Each group was randomly assigned to each experimental condition: three study groups and a Control group.

Before starting the study period, the parents or legal carers of the participants received a letter to inform them of the study process and purpose. They were asked to fill in and sign a consent form indicating their agreement for their children to be included in the study.

\subsection{Procedure}

Before starting the experiments $\left(t_{0}\right)$, each participating child (including those of the Control group) completed the baseline assessment Knowledge (Q1) and Habits (Q2) questionnaires.

Next, children in the study groups, i.e. With Role Switching (WRS), Without Role Switching (W/O $R S)$ and Video $(V)$ participated in the three different creative dance sessions $\left(s_{1}, s_{2}\right.$ and $\left.s_{3}\right)$ over a period of three consecutive weeks (one session per week). Each session lasted a maximum of 30 minutes.

During each encounter a child entered the room where the interactive area was set, accompanied by one of the researchers (Figure 4). The researcher turned on the camera and stayed behind the scene while the interaction took place. The child was told to follow the instructions the system would give and no intervention from the researcher occurred unless problems with the system arose. Once the session was over, the researcher took the child out of the room.

During the farewell of the first session, $t_{1}$, children in the study groups were invited by the robot or the interactive video to set two long term goals, one related to nutrition and one to physical activity, by signing a pact between them. Children in the Control group set these goals immediately after the baseline questionnaire at time $t_{0}$ (no pact in this case, but a commitment to pursue the objectives).

At the end of the third session, $t_{3}$, children in 


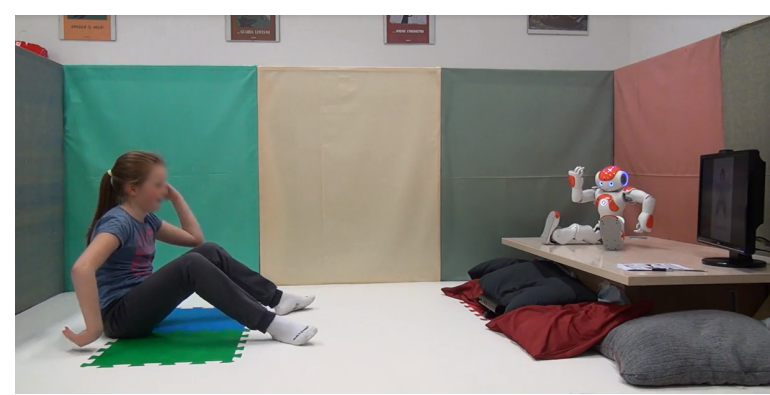

(a)

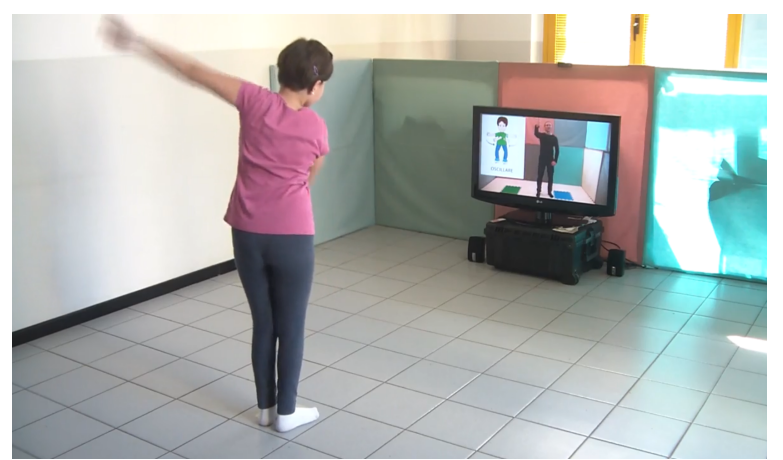

(b)

Figure 4: Area where the children interacted with system: (a) robot-base ( $W R S$ and $W / O R S$ experimental conditions) and (b) video-based ( $V$ experimental condition).

the study groups were also asked to fill in the Engagement questionnaire (Q4). Moreover, children received a sealed envelope to be delivered to their parents including the Behaviour Change questionnaire (Q3).

Finally, 10 days after the last session $\left(t_{4}\right)$, all children repeated Q1 and Q2 to assess the knowledge they had gained and possible changes in their habits. Q3 was collected at this point as well. Figure 5 summarises the experimental protocol for all groups.

\subsection{Hypothesis}

The goal of this work is to study whether an interactive robotic system can be used as a motivational support and learning tool. The rational behind this idea is that a social system that is capable of engaging children, during a certain period of time, will have an impact on children's awareness and goal achievement. Moreover, we evaluate two different systems (robot-based and video-based) and two different interactive approaches (role reversal and no role reversal) to analyse the different engagement mechanisms and their impact on the observed outcomes.

Thus, the following hypotheses are considered:

H1 Children exposed to the systems (WRS, $W / O$ $R S$ and $V$ groups) will be more aware of healthy habits, compared to those with no exposure to such systems ( $C$ group).

$\mathrm{H} 2$ Children exposed to the systems (WRS, W/O $R S$ and $V$ groups) will achieve a positive change in habits, in terms of diet and physical activity, over children belonging to the $C$ group.

H3 Children exposed to the robot-based systems (WRS, W/O RS groups) will show a higher motivational level towards changing habits over children exposed to the video-based system ( $V$ group).

H4 Children exposed to the robot-based system With Role Switching (WRS) will show a higher motivational level towards changing habits compared to those with no exposure to it $(W / O R S)$.

H5 Children exposed to the robot-based system with Role Switching (WRS) will show a higher engagement level in the interactive activity compared with children exposed to the other systems ( $W / O R S$ and $V$ groups). 
Preprint of paper to appear at the International Journal of Social Robotics (2016).

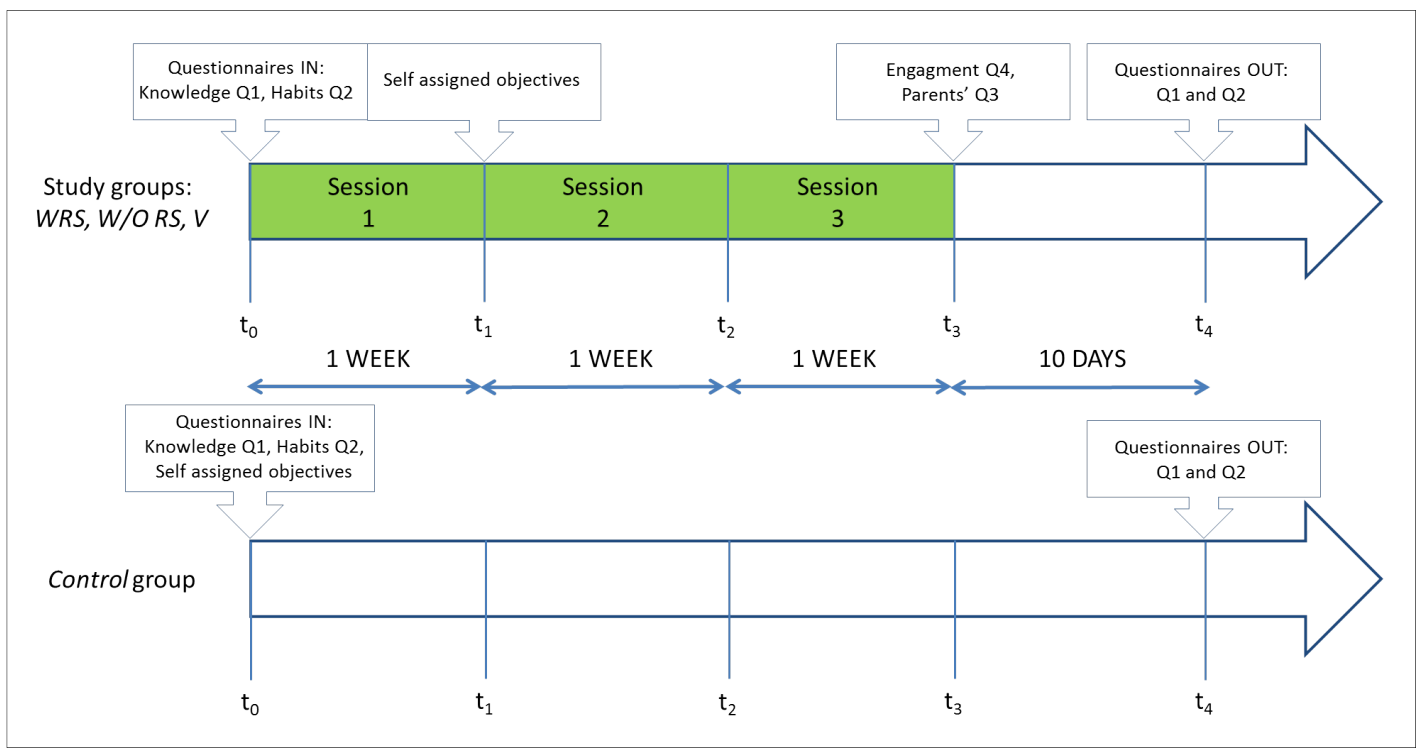

Figure 5: Experimental protocol time line.

\section{Results}

\subsection{Learning outcomes}

Children's answers on the Knowledge questionnaire (Q1) were rated on a 1-3 scale, where 3 corresponds to the maximum score. Table 3 summarises the average scores per question and experimental group, both at the beginning and at the end of the experiments.

A general knowledge improvement can be observed for all of the subjects involved in study, including the Control group. More specifically, participants in the WRS experimental condition showed a higher knowledge score compared to the other conditions, albeit without reaching statistical significance, and therefore, the first experimental Hypothesis H1 (children exposed to the systems will be more aware of healthy habits) cannot be verified.

\subsection{The motivational aspect}

Table 4 summarises the average scores obtained in the Habit questionnaire (Q2), comparing the pre (In) and post (Out) results. We can observe a trend towards improvement in the three study groups, i.e. WRS,
$W / O R S$ and $V$ and not in the Control group. This observation is also reinforced by the one-way ANOVA analysis applied: $F(3,308.95)=4.95, p<.01$, with a large effect size $\left(\omega^{2}=.13\right)$, confirming Hypothesis $\mathrm{H} 2$ and indicating that motivational support does have a positive impact on habit change. However, no clear patterns were found within the study groups: children improved their habits in a comparable way in all three conditions. Thus, we cannot validate $\mathrm{Hy}-$ pothesis $\mathrm{H} 4$.

On the other hand, clear evidence of a positive effect on habits has been found in the results from the children's self-assigned goals. In both the groups in which children interacted with the robot ( WRS, W/O $R S), 8$ out of 14 achieved at least one of their selfassigned goals; on the contrary, only 3 out of 13 succeeded in the Video group, and 3 out 43, in the Control one. The performed analysis corroborates the differences found (robot vs video: $\chi^{2}=4.14, P=.04$ for $p<.05$; robot vs control: $\chi^{2}=21.77, P=3 E-06$ for $p<.05)$ and we can therefore confirm Hypothesis H3.

This result is also reinforced by the analysis performed on the parents' questionnaire (Q3). Parents 
Preprint of paper to appear at the International Journal of Social Robotics (2016).

\begin{tabular}{c|c|c|c|c|c|c|c|c|c|c} 
& \multicolumn{2}{|c|}{ Q1.1 } & \multicolumn{2}{c|}{ Q1.2 } & \multicolumn{2}{c|}{ Q1.3 } & \multicolumn{2}{c}{ Q1.4 } & \multicolumn{2}{c}{ Q1.5 } \\
\cline { 2 - 11 } & In & Out & In & Out & In & Out & In & Out & In & Out \\
\hline \multirow{2}{*}{$W R S$} & 2.86 & 3.00 & 2.86 & 3.00 & 2.71 & 2.86 & 2.43 & 2.71 & 2.69 & 2.93 \\
& $(0.36)$ & $(0.00)$ & $(0.53)$ & $(0.00)$ & $(0.73)$ & $(0.53)$ & $(0.51)$ & $(0.47)$ & $(0.48)$ & $(0.27)$ \\
\hline \multirow{2}{*}{$W / O R S$} & 3.00 & 3.00 & 2.93 & 2.86 & 3.00 & 3.00 & 2.57 & 2.86 & 3.00 & 2.86 \\
& $(0.00)$ & $(0.00)$ & $(0.27)$ & $(0.36)$ & $(0.00)$ & $(0.00)$ & $(0.51)$ & $(0.36)$ & $(0.00)$ & $(0.36)$ \\
\hline \multirow{2}{*}{$V$} & 2.77 & 3.00 & 3.00 & 3.00 & 2.92 & 3.00 & 2.69 & 2.85 & 2.85 & 3.00 \\
& $(0.60)$ & $(0.00)$ & $(0.00)$ & $(0.00)$ & $(0.28)$ & $(0.00)$ & $(0.48)$ & $(0.38)$ & $(0.38)$ & $(0.00)$ \\
\hline \multirow{2}{*}{$C$} & 2.72 & 2.88 & 2.86 & 2.95 & 2.84 & 2.88 & 2.17 & 2.47 & 2.71 & 2.67 \\
& $(0.55)$ & $(0.33)$ & $(0.46)$ & $(0.21)$ & $(0.43)$ & $(0.39)$ & $(0.88)$ & $(0.80)$ & $(0.46)$ & $(0.47)$ \\
\hline
\end{tabular}

Table 3: Average and standard deviation (within brackets) scores before (IN) and after (OUT) the experiments for Knowledge Gain (questionnaire Q1) per condition and question. Values range from 1-3, where 3 corresponds to the maximum score.

\begin{tabular}{c|c|c|c|c|c|c|c|c|c|c|c|c} 
& \multicolumn{2}{|c|}{ Q2.1 $[4]$} & \multicolumn{2}{c}{ Q2.2 $[2]$} & \multicolumn{2}{c|}{ Q2.3 $[4]$} & \multicolumn{2}{c|}{ Q2.4 $[5]$} & \multicolumn{2}{c|}{ Q2.5 $[5]$} & \multicolumn{2}{c}{ Q2.6 $[4]$} \\
\cline { 2 - 13 } & In & Out & In & Out & In & Out & In & Out & In & Out & In & Out \\
\hline \multirow{2}{*}{$W R S$} & 3.57 & 3.71 & 1.57 & 1.57 & 2.50 & 2.50 & 3.07 & 3.36 & 3.21 & 3.29 & 2.50 & 3.21 \\
& $(1.05)$ & $(0.80)$ & $(0.49)$ & $(0.49)$ & $(1.24)$ & $(0.98)$ & $(0.59)$ & $(0.61)$ & $(0.77)$ & $(0.70)$ & $(0.91)$ & $(0.86)$ \\
\hline \multirow{2}{*}{$W / O R S$} & 3.43 & 3.50 & 1.50 & 1.79 & 3.29 & 3.43 & 3.29 & 3.43 & 3.29 & 3.43 & 2.85 & 3.14 \\
& $(0.98)$ & $(0.98)$ & $(0.50)$ & $(0.41)$ & $(0.88)$ & $(0.82)$ & $(0.59)$ & $(0.62)$ & $(0.59)$ & $(0.73)$ & $(0.95)$ & $(0.74)$ \\
\hline \multirow{2}{*}{$V$} & 3.54 & 3.62 & 1.46 & 1.77 & 3.08 & 3.15 & 3.00 & 3.31 & 3.00 & 3.31 & 2.62 & 3.08 \\
& $(0.93)$ & $(0.74)$ & $(0.50)$ & $(0.42)$ & $(1.00)$ & $(1.03)$ & $(0.68)$ & $(0.72)$ & $(0.39)$ & $(0.82)$ & $(0.74)$ & $(0.73)$ \\
\hline \multirow{2}{*}{$C$} & 3.42 & 3.21 & 1.56 & 1.49 & 2.36 & 2.14 & 3.26 & 3.23 & 3.05 & 3.07 & 2.93 & 2.93 \\
& $(1.04)$ & $(1.13)$ & $(0.50)$ & $(0.50)$ & $(1.21)$ & $(1.19)$ & $(0.69)$ & $(0.64)$ & $(0.81)$ & $(0.82)$ & $(0.92)$ & $(0.90)$ \\
\hline
\end{tabular}

Table 4: Average and standard deviation (within brackets) scores before (IN) and after (OUT) the experiments for Habit Change (questionnaire Q2) per condition and question. The maximum score per question is indicated in square brackets on the top row. 
Preprint of paper to appear at the International Journal of Social Robotics (2016).

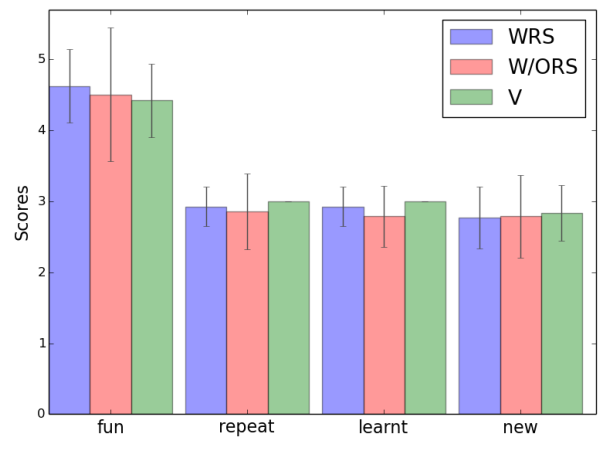

Figure 6: Average scores of the Engagement questionnaire (Q4) for each group. "Fun" is described on a $1-5$ scale, where 5 corresponds to "lots of fun". The remaining three questions are evaluated on a 1-3 scale, where 1 corresponds to "no", 2, to "maybe", and 3 , to "yes".

of children within the robot-based groups ( WRS and $W / O R S$ ) reported an improved behaviour towards healthy habits from their children. They indicated that during the study period, children were actually asking for more fruits and vegetables compared to children within the Video group $\left(\chi^{2}=5.92, p=.01\right)$.

\subsection{Activity engagement and robot perception}

No differences among groups were found in the Engagement questionnaire (Q4), and so far no claims can be made on Hypothesis H5. Figure 6 depicts the average scores of the children's responses. The interactive activity was enjoyed in general (fun) and children indicated that they would like to interact with the system again. (repeat). From their point of view, they did learn new things (learnt) and looked forward to learning new things with the system (new).

With respect to their perception of the robot, most children described it as being funny and smart, and attributed humanised descriptors to it (90\%), such as nice, tender and affectionate as opposed to engineering ones (10\%), such as mechanical and artificial as shown in Figure 7. Moreover, $61 \%$ of the children also perceived the robot as a friend, followed by a toy
(25\%), computer (10\%), and finally, as an adult (4\%), suggesting that children established a peer like bond with the robot. No clear differences between the two $W R S$ and $W / O R S$ study groups were found.

\subsection{Observed behavioural cues}

Analysing the results obtained from the video coding process of observed behavioural cues in the WRS and $W / O R S$ groups resulted into no statistical significance between the behavioural patterns of the two groups. However, we noted that children in the WRS group communicated acknowledgement (nodding the head for expressing a "yes" or "no" confirmation of what they are understanding) more often than children in the $W / O R S$ group (Figure 8a). Moreover, as observed in Figure 8b, the number of children who used non-verbal behaviours to communicate increased across sessions in the WRS group. Some children also used other non-verbal communicative acts such as approaching, greeting, pointing, excitement or expressing preferences (e.g. a child revealed happy or sad faces when observing the different types of food they should eat).

Communication via verbal behaviour decreased across sessions, as shown in Figure 9, suggesting a positive alignment towards the activity and robot's capabilities, where body language is prioritised and is the main means of communication. We can nevertheless observe that children in the WRS group were more open to communicate through verbal utterances, as a very large dispersion of expected verbal behaviour occurred in the $W / O R S$ compared to the $W R S$ group. Moreover, unexpected speech, such as greetings, acknowledging, asking and clarifying, took place much more often in the WRS than in the $W / O$ $R S$ group.

Figure 10a depicts the percentage of time children spent looking away from the robot. No statistical difference between the two groups was found. It is interesting to note that children in the WRS looked away more often than children in the $W / O R S$ group. This could be interpreted as a higher level of distraction. However, quite often we noticed that children in the WRS group would look towards the ceiling or to the side mainly while thinking what to propose next. 


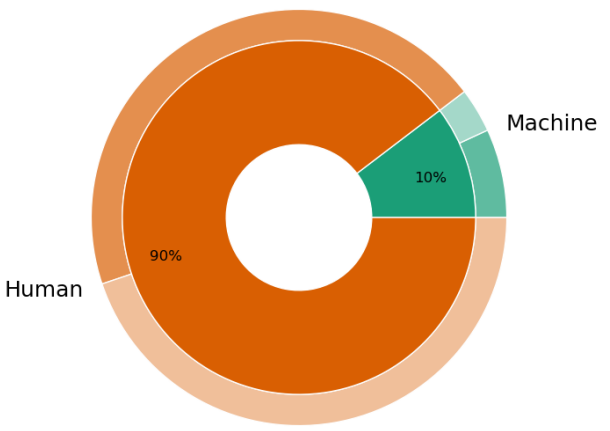

(a)

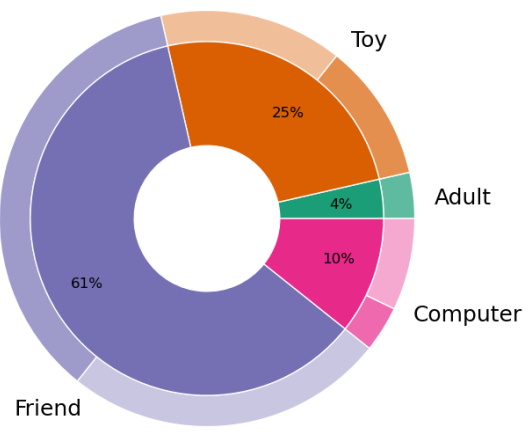

(b)

Figure 7: Robot's perception pie-charts based on (a) humanised vs. engineering adjectives and (b) relationship percentages. The outer circular crown describes the percentages based on experimental conditions: lighter colour corresponds to WRS group and darker colour, to W/O RS group.

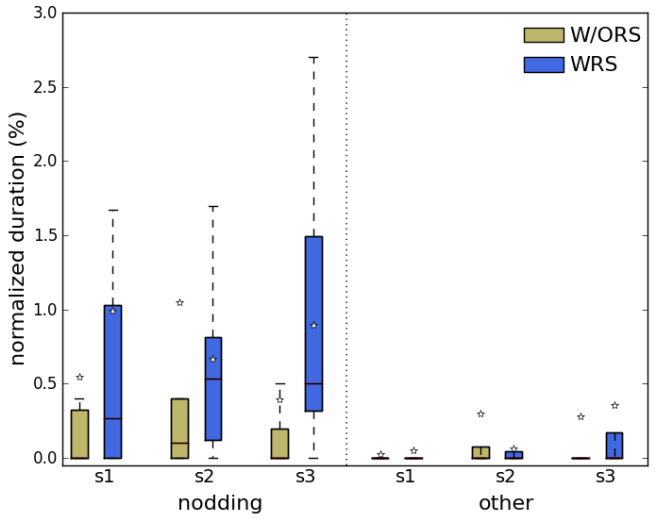

(a)

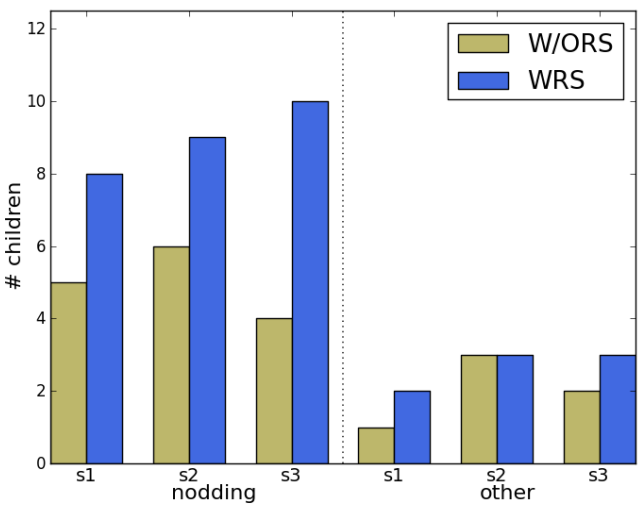

(b)

Figure 8: (a) Boxplot of the percentage of time invested in performing non-verbal behaviour to communicate with the robot per session (s1, s2 and s3 correspond to session 1,2 and 3 respectively) in both groups. The boxplots show the 75 and 25 percentile limits of the data, the median is represented with a horizontal line and the star corresponds to the average (this same format is present in all the boxplot figures presented in this work). (b) Number of children showing the non-verbal behaviours per session. 
Preprint of paper to appear at the International Journal of Social Robotics (2016).

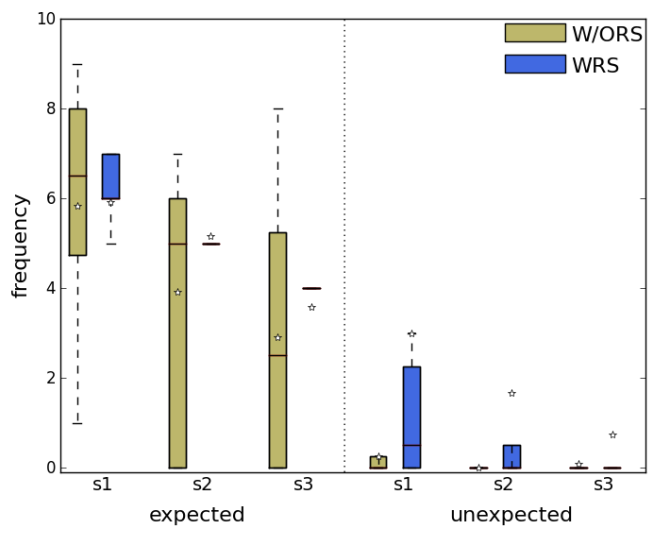

(a)

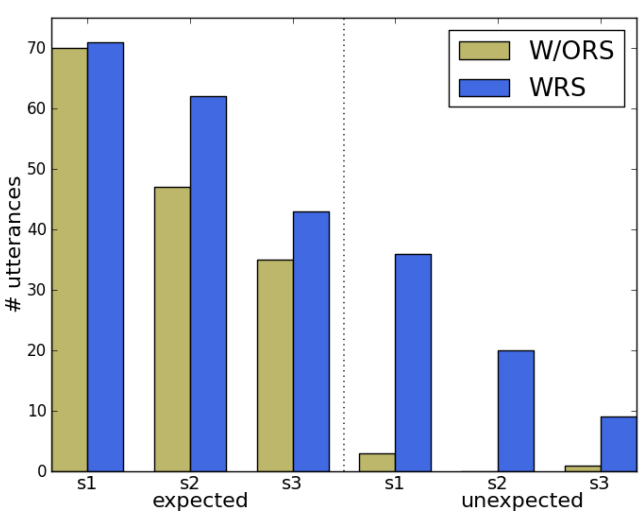

(b)

Figure 9: (a) Boxplot of the utterances frequency per sessions in both study groups. (b) Total number of utterances expressed per session.

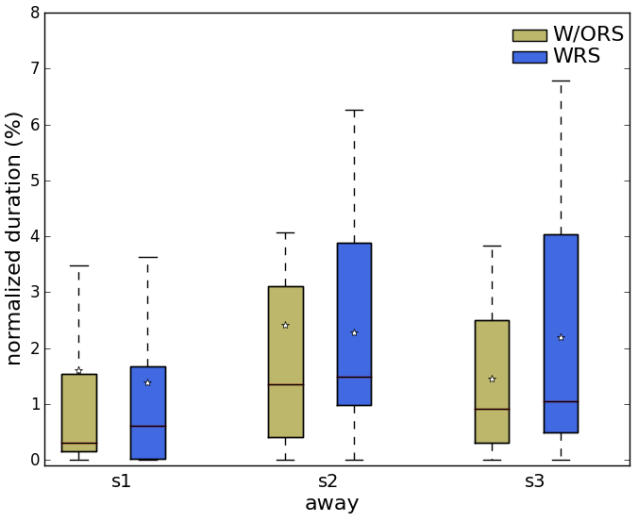

(a)

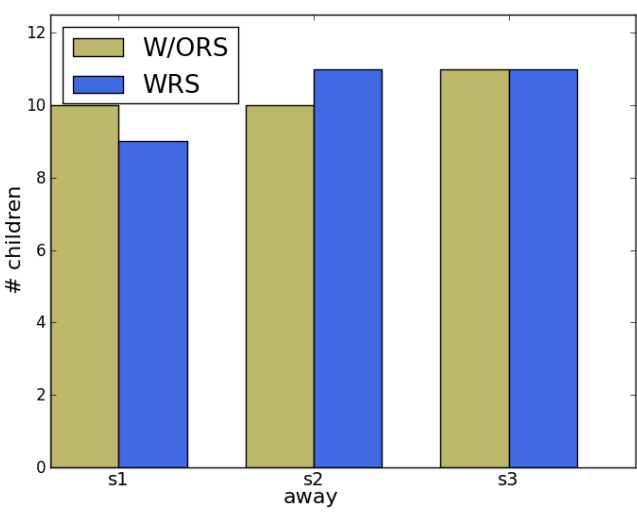

(b)

Figure 10: (a) Boxplot of the percentage of time that the children have spent looking away from the robot (or screen showing the concepts explained by the robot) per session in both groups. (b) Number of children looking away per session. 
Preprint of paper to appear at the International Journal of Social Robotics (2016).

6.4 Observed behavioural cues doi:10.1007/s12369-016-0356-9

This process only takes place in this group, where role switching takes place in the second half of the session and the child becomes the leader, indicating the robot what to do next, rather than following the robot.

No differences were found between the two groups in the task-related actions analysis (Figure 11a). However, from the purely qualitative point of view of the coders, children seemed to be more proactive in the WRS than in the $W / O R S$ group. Moreover, in both groups, we can observe that in session 2 children more actively participated during the travelling task compared to the other two sessions.

\subsubsection{PANAS evaluation}

In this section we present the results of the PANAS scale, analysed by implementing two between-within Factorial ANOVAs. The idea behind this analysis is to verify if there are any further insights that could support H5, relying on possible different behavioural changes across sessions per groups.

A good inter-coders agreement has been found for both the PANAS Positive and Negative Affect scale, with an average pairwise percent agreement of $72.2 \%$ and $70.4 \%$ respectively.

The Positive Affect scale and the Negative Affect one were used as Dependent Variables $(D V)$ in the two factorial models respectively. The within subject factor was the session (three sessions), repeated over time (one every week). The between subject factor consisted of the three experimental conditions in which the different samples of children were exposed to: W/O RS, WRS and Video groups.

All ratings on the PANAS scale consisted of a cumulative sum of the 5 items (positive or negative) followed by the averaged ratings of the three coders who observed the video recordings of the sessions. Minimum and maximum possible ratings ranged from 5 to 25 .

Planned Comparisons were used to test (based on H5) whether the WRS group differentiate from the other two groups. Thus, linear trends on the within subject factor were computed as Planned Contrasts. Both Planned Comparisons were applied in the two factorial experiments respectively.
Table 5 summarises the statistics obtained per session and experimental condition. A high evaluation of the Positive Affect is observed, specially for the $W R S$ group in all three sessions.

Omnibus Factorial ANOVA, $F(2,64)=.757, p>$ $.05 ; F(4,64)=.300, p>.05$ per session and group, and Planned Contrasts for the within subject factor (sessions over time) did not reveal any trend or difference $(p>.05)$, revealing a very low power (i.e., Power $=.17$ and .11 respectively).

Planned Contrasts on the experimental condition were very close to be significant with an Omnibus Contrast of $F(2,32)=3.236, p=.053$, with a small effect size (partial $\eta^{2}=.16$ and Power $=.58$ ). The $W R S$ condition significantly differed from the $W / O$ $R S$ condition (contrast $p<.02$ ), but not from the Video group (contrast $p>.05$ ). As shown in Figure 12a, the WRS group had an effect on the children with respect to other groups (see Section 7 for more details). No interactions across the group and sessions were detected.

Descriptive statistics for the Negative Affect scale are reported in Table 6 . We can observe a tendency towards less negative evaluations as the encounters between children and the tutor progress from session 1 to 3 , especially in the $W / O R S$ and Video groups.

Omnibus factorial ANOVA for the repeated measures sessions was significant $F(1.655,64)=$ $6.735, p<.01$ with a small effect size (partial $\eta^{2}=$ .17 and Power $=.86)$. The experimental conditions and sessions were not interacting: $F(3.311,64)=$ $0.322, p>.05$ (partial $\eta^{2}=.02$ and Power $\left.=.11\right)$.

The Planned Contrasts for the sessions over time did reveal that session 1 differed in the Negative Affect scale compared to session 2 (contrast $p<.05$ ) and session 3 (contrast $p<.05$ ). These Planned contrasts were significant with a small effect size (partial $\eta^{2}=.15$ and $=.23$ respectively; the Power is $=.64$ and $=.85$ respectively).

A clear downward trend with less negative evaluations at session 3 was detected as shown in Figure 12b. However, Planned Contrasts on the experimental condition revealed no significant difference on the Negative Affect scale (all Planned Contrasts $p>.05)$. 
Preprint of paper to appear at the International Journal of Social Robotics (2016).

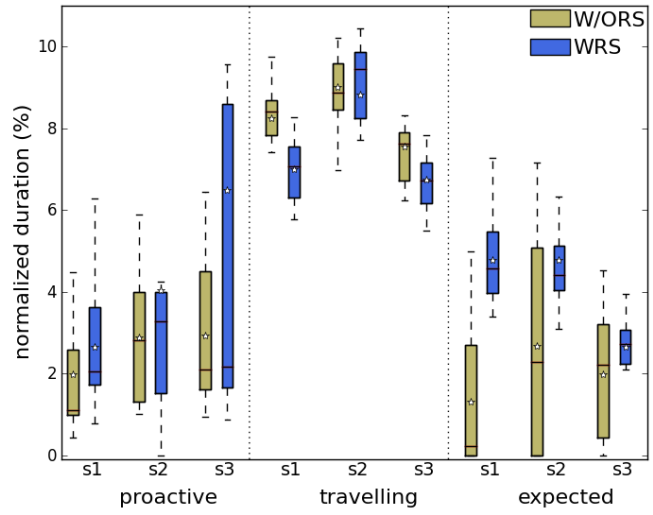

(a)

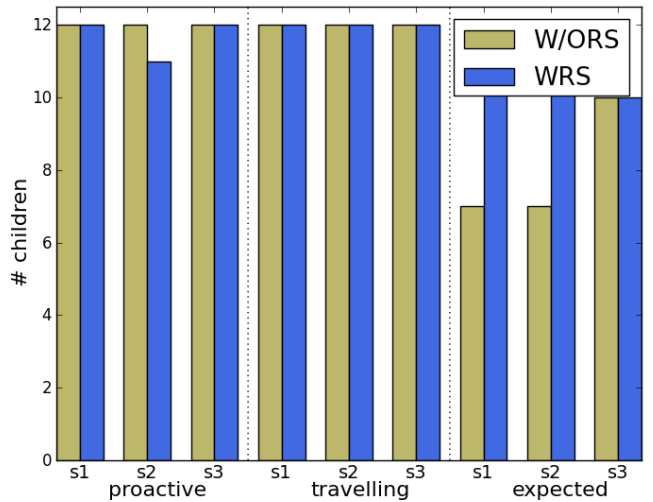

(b)

Figure 11: (a) Boxplot of the percentage of time that the children performed different types of actions related to the dance activity task during the three sessions. (b) Number of children showing task related actions per session.

\begin{tabular}{c|c|cc|cc|cc}
\multirow{2}{*}{ Condition } & \multirow{2}{*}{$\mathrm{N}$} & \multicolumn{2}{|c|}{ Session 1 } & \multicolumn{2}{c|}{ Session 2 } & \multicolumn{2}{c}{ Session 3 } \\
\cline { 3 - 8 } & & $\mu$ & $\sigma$ & $\mu$ & $\sigma$ & $\mu$ & $\sigma$ \\
\hline$W / O R S$ & 12 & 17.83 & 2.59 & 18.42 & 2.08 & 17.47 & 1.89 \\
$\boldsymbol{W R} \boldsymbol{S}$ & 11 & $\mathbf{1 9 . 5 1}$ & 1.77 & $\mathbf{1 9 . 1 2}$ & 1.99 & $\mathbf{1 9 . 2 1}$ & 1.78 \\
$V$ & 12 & 18.22 & 2.40 & 18.94 & 3.12 & 17.81 & 2.33 \\
\hline
\end{tabular}

Table 5: Statistics on Positive Affect (i.e.: evaluated average ratings scores) per session and condition.

\begin{tabular}{c|c|cc|cc|cc}
\multirow{2}{*}{ Condition } & \multirow{2}{*}{$\mathrm{N}$} & \multicolumn{2}{|c|}{ Session 1 } & \multicolumn{2}{|c|}{ Session 2 } & \multicolumn{2}{c}{ Session 3 } \\
\cline { 3 - 8 } & & $\mu$ & $\sigma$ & $\mu$ & $\sigma$ & $\mu$ & $\sigma$ \\
\hline$W / O R S$ & 12 & 7.03 & 1.34 & 6.41 & 1.05 & 5.94 & 0.80 \\
$W R S$ & 11 & 6.70 & 1.33 & 6.12 & 1.01 & 6.09 & 1.01 \\
$V$ & 12 & 7.08 & 2.37 & 6.08 & 1.23 & 5.75 & 0.78 \\
\hline
\end{tabular}

Table 6: Statistics on Negative Affect (i.e.: evaluated average ratings scores) per session and condition. 


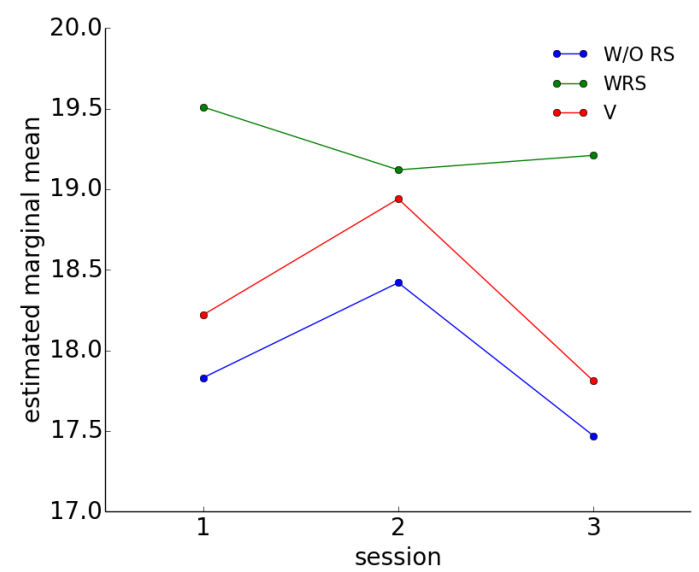

(a)

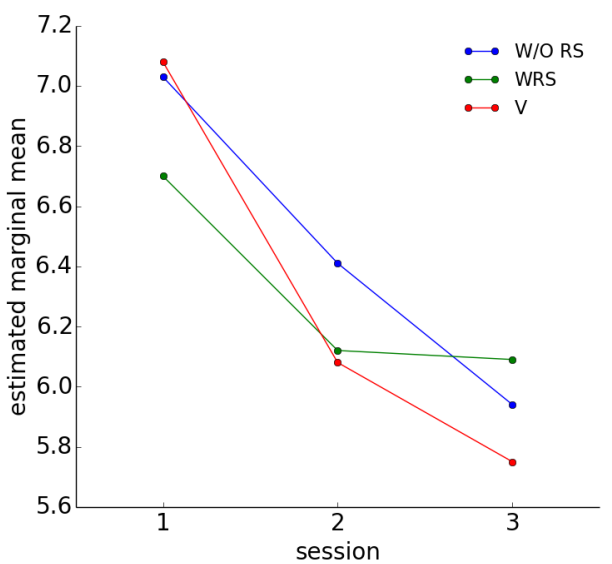

(b)

Figure 12: Trend plot of PANAS (a) Positive and (b) Negative Affect.

\section{Discussion}

The main goal of this work is to study the impact of motivational mechanisms in child-robot interaction and their effect on the adoption of healthy life-styles. A secondary goal is to compare these impacts while using different technologies, i.e. robots vs. interactive videos. Moreover, an underlying challenge is to assess the interactions in familiar environments for children, such as their own school classrooms. Our goal is to move away from laboratories or artificial environments, unknown to children and potentially frightening to them at first.

In order to achieve conclusive results for all the analysed variables, a sample size exceeding that of 84 children we used in this study would have been ideal. However, recruiting a large number of children from public organisations remains a big challenge: getting consent from parents or tutors and complying to school policies remains difficult. Although we are aware of the sample size limitations in this study, the results are promising enough to warrant further investigation.

\subsection{Learning outcomes}

From the analysis of the results, no clear impact on learning gains was achieved in any of the groups, contradicting Hypothesis H1. However, a general improvement can be observed in all the children, including those belonging to the Control group. This result suggests that children in the Control group could be self-motivated in learning about healthy habits after fulfilling the pre-experiment questionnaire. Children could feel more curious about the questions and be encouraged to inform themselves through other sources (e.g. family, books, etc.), but not at the school $^{8}$. A highly controlled environment would be more suitable to compare experimental conditions against controlled ones. However our key interest is to explore the use of robot in real world scenarios where children interact with them in natural environments. Further experiments extending the study to also monitor the children's social environment (e.g. talking to parents/caregivers) would be useful in order to understand whether children seek alternative

\footnotetext{
${ }^{8}$ As already mentioned in the study design, teachers confirmed that children were not having any lessons regarding healthy life styles during the study period.
} 
Preprint of paper to appear at the International Journal of Social Robotics (2016).

sources of information on their own.

With regards to the lack of evidence for knowledge gained in the study groups, we believe that the number of sessions, and therefore, repetition of information, was not long enough for children to capture and absorb new concepts. The proposed framework is designed as a support tool for teachers, and not as a single source of learning. Therefore, in an ideal scenario, children would learn new ideas with school teachers and then practice and reinforce the knowledge acquired with the system.

Moreover, similar findings where reported in [17], where the authors reported that children interacting with a social robot achieved lower knowledge gains compared to alternative conditions (i.e. an asocial robot and a screen-based system). They suggest that the reasons for such unexpected findings are that children were either (a) paying more attention to the social behaviour instead of the sessions content or (b) that the complex behaviour of the robot resulted in a cognitive overload for children inhibiting their capacity to process the information. Additional research on these lines should be conducted to confirm these ideas.

\subsection{Motivational aspect}

We confirmed the motivational impact of innovative approaches on children (Hypothesis H2). Moreover, although the content of the interactive video and robot systems was exactly the same (both in terms of theoretical concepts and motivational cues), the robot-based systems were more effective motivators towards the adoption of healthier habits (supporting Hypothesis H3).

Based on this evidence, we can conclude that the use of robots as support tools in order to motivate and promote the acquisition of good habits in children is an effective strategy. We believe that the combination of a robotic character, with whom children felt committed to pursue their self-assigned goals, and an engaging and fun environment, as the one of creative dance, can lead to positive results in the long term.

Contrary to our expectations, Hypothesis H4 was not confirmed, suggesting that intrinsic motivation to achieve the self-assigned goals is not influenced by the role-switching approach studied in this work. One possible reason for not finding significant differences between the impact of using or not using roleswitching as an intrinsic motivational mechanism is that the educational framework proposed is powerful enough on its own to motivate and engage children. Thus, the effect of an additional motivational mechanism is diluted within this context. A motivational mechanism might have higher influence in other contexts, where the task is not as engaging and motivating as the one proposed. We thus foresee the need of investigating the role-switching mechanism in simpler environments to evidence its effectiveness.

\subsection{Activity engagement}

Different tools were used to investigate children's level of engagement: $(i)$ a self-assessment questionnaire (Q4); (ii) video coding analysis on behavioural cues; and (iii) the PANAS study.

No statistical evidence could be found to confirm that children interacting with a robot would achieve higher levels of engagement compared to those interacting with an interactive video (H5). However, we could observe the following trends comparing children belonging to the $W R S$ condition against children in the $W / O R S$ condition:

- Higher non-verbal communication and lower verbal communication, suggesting an alignment towards the activity and the robot's capabilities, in which body language is prioritised (i.e. children could infer that the communication with the robot was mainly done through body movement and not through verbal utterances);

- Higher pro-activeness of actions in the sessions, which in turn could reflect higher creativity and involvement in the activity.

Moreover, from a qualitative point of view, all researchers performing the video coding expressed their impression of higher level of engagement in children belonging to the WRS group than in the other conditions. 
Preprint of paper to appear at the International Journal of Social Robotics (2016).

doi:10.1007/s12369-016-0356-9

Concerning the PANAS scale analysis, it is worth mentioning that these observations should be considered as contextual only because of the very small differences among the study groups. Overall, ratings in the PANAS Negative Affect analysis were less negative across the sessions in all the study groups (see Figure 12b) suggesting that children could feel more confident and at ease over time with the systems and the activity proposed. In particular this trend revealed to be significantly supported by statistics for the $W / O R S$ and Video groups, but not for the WRS one, which tendency remained more stable over time.

This observation is also reflected in the PANAS Positive Affect scale analysis, in which the WRS group outcomes were more stable over time in contrast to higher variations for the other groups (see Figure 12a). In addition to that, a significant difference was found between the Positive scale evaluations of the two robot-based groups: those of the $W R S$ study condition were higher than those of the $W / O R S$ one. The combination of these insights reinforces the coders' general impression that children exposed to the WRS system seemed to be more active and engaged compared to children in the other groups. Further investigations are therefore needed to validate the effectiveness of the role-switching approach as a determinant factor to reach a sustained engagement level in a long term perspective.

It is also worth mentioning that the highest levels of Positive Affect in the $W / O R S$ and Video groups were observed in session 2 , which corresponds to the most active session (including jumps and quick movements). Children tend to have a preference towards these type of movements (as we already observed in [35]) and therefore feel more engaged. This suggests a correlation with higher perception of Positive Affect. The influence of activities/tasks on engagement management should be further investigated as they could be used as mechanisms to capture the user's interest when distractions or lost of interest are detected.

\section{Conclusions}

In the current work we describe the study of an intrinsic motivational mechanism, targeted to foster the acquisition of knowledge and the promotion of healthy habits in school age children. More precisely, we propose a role-switching mechanism (similar to that of active learning) centred around a dance activity. Children alternate the leading role with a humanoid robot as part of the development of the activity sessions. By doing so, children can feel in control of the session becoming the activity tutors, while the robot becomes the apprentice (i.e. the subject under evaluation). As a consequence, children should be highly engaged in the task proposed and intrinsic motivation should prove more effective.

We performed a large set of experiments in Italy, involving 84 children, from which 41 interacted with the proposed approach in three sessions over three weeks. The remaining 43 corresponded to the baseline group to compare the evaluations. A wide set of measures were considered, including both quantitative and qualitative evaluations. The experiments were evaluated along three main areas: awareness, habit change and level of engagement during the interaction. Five hypotheses were presented, from which three were confirmed, one was partially confirmed and one was not confirmed. Further evaluation should be done to achieve conclusive results. More precisely, the system has proved its effectiveness as a motivational tool to achieve habit change and to promote engagement in task execution. As a future work, we believe that long-term interaction should be studied over longer periods of time in order to achieve conclusive evidence supporting the remaining multiple hypotheses presented in this research.

\section{9 acknowledgements}

The work was supported in part by the EU FP7 ALIZ-E project, grant No. ICT-248116, and EU H2020 project PAL, grant H2020-PHC-643783. We wish to thank Sara Bellini, Monica Verga and Marco Mosconi for being a key part of this experience as well as the children, their families and teachers who 
Preprint of paper to appear at the International Journal of Social Robotics (2016).

supported us and participated enthusiastically in this research.

\section{References}

[1] Ruth S. Aylett, Ginevra Castellano, Bogdan Raducanu, Ana Paiva, and Mark Hanheide. Longterm socially perceptive and interactive robot companions: challenges and future perspectives. In Proc. of Int. Conf. on Multimodal Interfaces, pages 323-326, 2011.

[2] I. Baroni, M. Nalin, M. Coti Zelati, E. Oleari, and A. Sanna. Designing motivational robot: How robots might motivate children to eat fruits and vegetables. In Proceedings of the 23rd IEEE International Symposium on Robot and Human Interactive Communication (RoMAN), pages 796-801. IEEE, 2014.

[3] Tony Belpaeme, Paul Baxter, Joachim de Greeff, James Kennedy, Robin Read, Rosemarijn Looije, Mark Neerincx, Ilaria Baroni, and MattiaCoti Zelati. Child-robot interaction: Perspectives and challenges. In Guido Herrmann, MartinJ. Pearson, Alexander Lenz, Paul Bremner, Adam Spiers, and Ute Leonards, editors, Social Robotics, volume 8239 of Lecture Notes in Computer Science, pages 452-459. Springer International Publishing, 2013.

[4] Tony Belpaeme, Paul E Baxter, Robin Read, Rachel Wood, Heriberto Cuayáhuitl, Bernd Kiefer, Stefania Racioppa, Ivana KruijffKorbayová, Georgios Athanasopoulos, Valentin Enescu, et al. Multimodal child-robot interaction: Building social bonds. Journal of HumanRobot Interaction, 1(2):33-53, 2012.

[5] Nadia Bianchi-Berthouze. Understanding the role of body movement in player engagement. Human-Computer Interaction, 28(1):40$75,2013$.

[6] Timothy W. Bickmore and Rosalind W. Picard. Establishing and maintaining long-term human- computer relationships. Transactions on Computer Human Interaction, 12(2):293-327, 2005.

[7] Cynthia L Breazeal. Designing sociable robots. MIT press, 2004.

[8] Allison Bruce, Illah Nourbakhsh, and Reid Simmons. The role of expressiveness and attention in human-robot interaction. In Robotics and Automation, 2002. Proceedings. ICRA'02. IEEE International Conference on, volume 4, pages 4138-4142. IEEE, 2002.

[9] Martyn Cooper, David Keating, William Harwin, and Kerstin Dautenhahn. Robots in the classroom-tools for accessible education. Assistive technology on the threshold of the new millennium, pages 448-452, 1999.

[10] European Food Information Council. Foodbased dietary guidelines in europe, 2009. http://www.eufic.org/article/en/expid/foodbased-dietary-guidelines-in-europe/.

[11] Kerstin Dautenhahn. Socially intelligent robots: dimensions of human-robot interaction. Philosophical Transactions of the Royal Society B: Biological Sciences, 362(1480):679-704, 2007.

[12] Juan Fasola and Maja J Mataric. Robot motivator: Increasing user enjoyment and performance on a physical/cognitive task. In Development and Learning (ICDL), 2010 IEEE 9th International Conference on, pages 274-279. IEEE, 2010.

[13] Terrence Fong, Illah Nourbakhsh, and Kerstin Dautenhahn. A survey of socially interactive robots. Robotics and autonomous systems, 42(3):143-166, 2003.

[14] Rachel Gockley, Allison Bruce, Jodi Forlizzi, Marek Michalowski, Anne Mundell, Stephanie Rosenthal, Brennan Sellner, Reid Simmons, Kevin Snipes, Alan C Schultz, et al. Designing robots for long-term social interaction. In IEEE/RSJ International Conference on Intelligent Robots and Systems (IROS), pages 13381343. IEEE, 2005. 
[15] Libby Hanna, Kirsten Risden, and Kirsten Alexander. Guidelines for usability testing with children. Interactions, 4(5):9-14, 1997.

[16] Takayuki Kanda, Rumi Sato, Naoki Saiwaki, and Hiroshi Ishiguro. A two-month field trial in an elementary school for long-term humanrobot interaction. Robotics, IEEE Transactions on, 23(5):962-971, 2007.

[17] James Kennedy, Paul Baxter, and Tony Belpaeme. The robot who tried too hard: Social behaviour of a robot tutor can negatively affect child learning. In Proceedings of the Tenth Annual ACM/IEEE International Conference on Human-Robot Interaction, pages 67-74. ACM, 2015.

[18] Cory D Kidd and Cynthia Breazeal. A robotic weight loss coach. In Proceedings of the 22nd national conference on Artificial Intelligence, volume 2, pages 1985-1986. AAAI Press, 2007.

[19] Cory D Kidd and Cynthia Breazeal. Robots at home: Understanding long-term human-robot interaction. In IEEE/RSJ International Conference on Intelligent Robots and Systems (IROS), pages 3230-3235. IEEE, 2008.

[20] Jeff Laurent, Salvatore J. Catanzaro, Thomas E. Joiner Jr., Karen D. Rudolph, Kirsten I. Potter, Sharon Lambert, Lori Osborne, and Tamara Gathright. A measure of positive and negative affect for children: Scale development and preliminary validation. Psychological Assessment, 11(3):326-338, 1999.

[21] Min Kyung Lee, Sara Kiesler, and Jodi Forlizzi. Receptionist or information kiosk: How do people talk with a robot? In Proc. of Conf. on Computer supported cooperative work, pages 3140, 2010.

[22] I. Leite, C. Martinho, A. Pereira, and A. Paiva. As time goes by: Long-term evaluation of social presence in robotic companions. In Int. Symposium on Robot and Human Interactive Communication, pages 669-674, 2009.
[23] Iolanda Leite, Carlos Martinho, and Ana Paiva. Social robots for long-term interaction: A survey. Int. Journal of Social Robotics, pages 1-18, 2013.

[24] Iolanda Leite, André Pereira, Ginevra Castellano, Samuel Mascarenhas, Carlos Martinho, and Ana Paiva. Social robots in learning environments: a case study of an empathic chess companion. In Proc. of the Int. WS on Personalization Approaches in Learning Environments, volume 732, pages 8-12, 2011.

[25] M. Lewis and L Cañamero. An affective autonomous robot toddler to support the development of self-efficacy in diabetic children. In Proceedings of the 23rd Annual IEEE International Symposium on Robot and Human Interactive Communication (RO-MAN), pages 359364. IEEE, 2014.

[26] Stephen Paul Linder, Brian Edward Nestrick, Symen Mulders, and Catherine Leah Lavelle. Facilitating active learning with inexpensive mobile robots. Journal of Computing Sciences in Colleges, 16(4):21-33, 2001.

[27] Panos Markopoulos, Janet Read, Stuart MacFarlane, and Johanna Hoysniemi. Evaluating Children's Interactive Products: Principles and Practices for Interaction Designers. Morgan Kaufmann, 2008.

[28] Victor Ng-Thow-Hing, Ravi Kiran Sarvadevabhatla, and Sandra Okita. The learning with kids project: Retrospective and status report. In WS on human-robot interaction: perspectives and contributions to robotics from the human sciences, 2011.

[29] Office of Disease Prevention and Health Promotion. Physical activity guidelines, 2015. http://www.health.gov/paguidelines/.

[30] Sandra Y. Okita and Victor Ng-Thow-Hing. Learning together: Asimo developing an interactive learning partnership with children. In Int. Symposium on Robot and Human Interactive Communication, pages 1125-1130, 2009. 
Preprint of paper to appear at the International Journal of Social Robotics (2016).

[31] World Health Organization. A healthy lifestyle, 2015. http://www.euro.who.int/en/healthtopics/disease-prevention/nutrition/a-healthylifestyle.

[32] Sunghyun Park, Lilia Moshkina, and Ronald C. Arkin. Recognizing nonverbal affective behavior in humanoid robots. Intelligent Autonomous Systems 11: IAS-11, pages 12-21, 2010.

[33] B Robins, K Dautenhahn, R Te Boekhorst, and A Billard. Effects of repeated exposure to a humanoid robot on children with autism. In $D e$ signing a more inclusive world, pages 225-236. Springer, 2004.

[34] Ben Robins and Kerstin Dautenhahn. Developing play scenarios for tactile interaction with a humanoid robot: A case study exploration with children with autism. In Social Robotics, volume 6414 of Lecture Notes in Computer Science, pages 243-252. Springer Berlin Heidelberg, 2010.

[35] Raquel Ros, Ilaria Baroni, and Yiannis Demiris. Adaptive human-robot interaction in sensorimotor task instruction: From human to robot dance tutors. Robotics and Autonomous Systems, 62(6):707 - 720, 2014.

[36] Raquel Ros and Yiannis Demiris. Creative dance: An approach for social interaction between robots and children. In AlbertAli Salah, Hayley Hung, Oya Aran, and Hatice Gunes, editors, Human Behavior Understanding, volume 8212 of Lecture Notes in Computer Science, pages 40-51. Springer International Publishing, 2013.

[37] Richard M. Ryan and Edward L. Deci. Intrinsic and extrinsic motivations: Classic definitions and new directions. Contemporary Educational Psychology, 25(1):54 - 67, 2000.

[38] Selma Sabanovic, Marek Piotr Michalowski, and Linnda R. Caporael. Making friends: Building social robots through interdisciplinary collaboration. In Multidisciplinary Collaboration for Socially Assistive Robotics: Papers from the 2007
AAAI Spring Symposium, Technical Report SS07-0\%, pages 71-77. AAAI, 2007.

[39] Brian Scassellati. Imitation and mechanisms of joint attention: A developmental structure for building social skills on a humanoid robot. In ChrystopherL. Nehaniv, editor, Computation for Metaphors, Analogy, and Agents, volume 1562 of Lecture Notes in Computer Science, pages 176-195. Springer Berlin Heidelberg, 1999.

[40] J. M. Smith-Autard. The Art of Dance in Education. A\&C Black Publishers, 2002.

[41] F. Tanaka, A. Cicourel, and J. R. Movellan. Socialization between toddlers and robots at an early childhood education center. Proc. of the National Academy of Sciences, 104(46):1795417958, 2007.

[42] Fumihide Tanaka and Shizuko Matsuzoe. Children teach a care-receiving robot to promote their learning: Field experiments at a classroom for vocabulary learning. Journal of HumanRobot Interaction, 1(1):78-95, 2012.

[43] Fumihide Tanaka, Javier R Movellan, Bret Fortenberry, and Kazuki Aisaka. Daily hri evaluation at a classroom environment: reports from dance interaction experiments. In Proceedings of the 1st ACM SIGCHI/SIGART conference on Human-robot interaction, pages 3-9. ACM, 2006 .

[44] Richard J Vallerand and Greg Reid. On the causal effects of perceived competence on intrinsic motivation: A test of cognitive evaluation theory. Journal of Sport Psychology, 6(1):94$102,1984$.

[45] Kazuyoshi Wada and Takanori Shibata. Robot therapy in a care house-change of relationship among the residents and seal robot during a 2month long study. In Robot and Human interactive Communication, 2007. RO-MAN 200\%. The 16th IEEE International Symposium on, pages 107-112. IEEE, 2007. 
Preprint of paper to appear at the International Journal of Social Robotics (2016).

doi:10.1007/s12369-016-0356-9

[46] Kazuyoshi Wada, Takanori Shibata, Toshimitsu Q1.3 What should we do daily?

Musha, and Shin Kimura. Effects of robot therapy for demented patients evaluated by eeg. In IEEE/RSJ International Conference on Intelligent Robots and Systems (IROS), pages 15521557. IEEE, 2005.

[47] Kazuyoshi Wada, Takanori Shibata, Toshimitsu Musha, and Shin Kimura. Robot therapy for elders affected by dementia. Engineering in Medicine and Biology Magazine, IEEE, 27(4):53-60, 2008.

[48] Peter Wittenburg, Hennie Brugman, Albert Russel, Alex Klassmann, and Han Sloetjes. ELAN: a professional framework for multimodality research. In Proceedings of Fifth International Conference on Language Resources and Evaluation, volume 2006, pages 1556-1559, 2006.

\section{Appendix}

The following questionnaires were provided to the participating children during the study period. The formatting has been changed: the originals used images combined with the answers in multiple choice questions and emoticons in the Likert-scale responses to make them more accessible for children. The original questionnaires were given in Italian. The following questionnaires are a translation of these.

\subsection{Knowledge Questionnaire (Q1)}

Q1.1 Watching TV, using a PC and playing videogames are activities that we must:

- Try to avoid

- Try to do everyday

- Do daily, also several times a day

Q1.2 Fruits and vegetables are food that we can eat:

- Daily, also several times a day

- Few times a week

- Rarely, and better try not to eat them
- Walk, use the stairs, being physically active

- Play videogames

- Eat very savory snacks and sweet snacks

Q1.4 Which food can we eat many times a week?

- Legumes

- Cold cuts

- Eggs

Q1.5 Which beverage can we daily drink?

- Milk

- Energy drink

- Sugary drink (like cold tea, soft drinks,...)

\subsection{Habit Questionnaire (Q2)}

Q2.1 What do you usually drink?

- Water

- Coke

- Orange juice

- Cold tea

- Milk

Q2.2 How many servings of fruit do you eat per day?

- Less than 1 in a day

-1 or 2 in a day

-3 or 4 in a day

-5 or more

Q2.3 How many servings of vegetables do you eat per day?

- Less than 1 time in a day

-1 or 2 in a day

- 3 or 4 in a day

-5 or more

Q2.4 How many times do you eat savory or sweet snacks? 
Preprint of paper to appear at the International Journal of Social Robotics (2016).

- Less than 1 time in a week

- 1-2 times a week

- 4-5 times a week

- Daily or several times a day

Q2.5 Which activity do you usually do in your free time?

- Watch TV

- Play at the pc

- Play sport

- Go to the park

- Other, specify:

Q2.6 If you play with a PC, for how long do you do it?

- Less than 1 hour

- Between 1 or 2 hours

- More than 2 hours

- I don't play with a PC

\subsection{Parents Questionnaire (Q3)}

1. Has your son/daughter spoken spontaneously about the activity done?

2. Has your son/daughter given more attention towards the food in the last week?

3. Has your son/daughter requested more fruits or vegetables in the last week?

4. Has your son/daughter asked you to modify something in HIS/HER food habit?

5. Has your son/daughter asked you information on food composition?

6. Has your son/daughter asked you to modify something in YOUR food habit?

7. Has your son/daughter done more physical activity than the usual (for example walk to school, use the stairs, ride a bicycle, etc.)?

8. Has your son/daughter proposed you to do some physical exercise together (for example walk instead of using the car, use the stairs instead of the lift, etc.)?

\subsection{Engagement Questionnaire (Q4)}

The questions are reported for both the robot and video study groups. Those highlighted with $(\star)$ were designed and given only to the children interacting with the robot.

1. How fun was the activity with $[\mathrm{Nao} /$ the interactive video]?

- Not fun at all 중

- Not that fun $(-)$

- Neutral $:$

- Good fun :)

- Great fun (2)

2. Would you like to play again with $[\mathrm{Nao} /$ the interactive video]?

- Yes

- No

- Maybe

3. ( $\star$ How much did you like Nao?

- Not at all $: 2$

- Not so much $)$

- Neutral $\odot$

- A lot $\odot$

- I loved it :-

4. ( $\star$ In your opinion, Nao seems more like...

- a friend

- a pet

- an adult

- a toy

- a computer

5. Did understand what [ $\mathrm{Nao} /$ the interactive $]$ video was requesting from you?

- Yes

- No

- Sometimes 
Preprint of paper to appear at the International Journal of Social Robotics (2016).

10.4 Engagement Questionnaire (Q4)doi:10.1007/s12369-016-0356-9

6. Do you think you've learned something new with [Nao/the interactive video]?

- Yes

- No

- Maybe

7. Would you like to learn something else with $[\mathrm{Nao} /$ the interactive video $]$ ?

- Yes

- No

- Maybe

8. If yes, what would you like to learn with [Nao/the interactive video]?

9. $(\star)$ Choose the word that in your opinion best describes Nao:

- mechanical

- kind

- fun

- smart

- fake

- fragile

- tender

- lovely 